\title{
BRANCHPOINT COVERING THEOREMS FOR CONFLUENT AND WEAKLY CONFLUENT MAPS
}

\author{
C. A. EBERHART, J. B. FUGATE, AND G. R. GORDH, JR.
}

\begin{abstract}
A branchpoint of a compactum $X$ is a point which is the vertex of a simple triod in $X$. A surjective map $f: X \rightarrow Y$ is said to cover the branchpoints of $Y$ if each branchpoint in $Y$ is the image of some branchpoint in $X$. If every map in a class $\mathscr{F}$ of maps on a class of compacta $C$ covers the branchpoints of its image, then it is said that the branchpoint covering property holds for $\mathscr{F}$ on $C$.

According to Whyburn's classical theorem on the lifting of dendrites, the branchpoint covering property holds for light open maps on arbitrary compacta. In this paper it is shown that the branchpoint covering property holds for (1) light confluent maps on arbitrary compacta, (2) confluent maps on hereditarily arcwise connected compacta, and (3) weakly confluent maps on hereditarily locally connected continua having closed sets of branchpoints. It follows that the weakly confluent image of a graph is a graph.
\end{abstract}

By a branchpoint of a compactum (i.e., compact metric space) $X$ we mean a point $p$ of $X$ which is the vertex of a simple triod lying in $X$. Given a surjective map $f: X \rightarrow Y$ between compacta we will say that $f$ covers the branchpoints of $Y$ provided each branchpoint of $Y$ is the image under $f$ of a branchpoint of $X$. Of course, in general, $f$ will not cover the branchpoints of its image, but if it does whenever $f$ is required to be in a particular class $\mathscr{F}$ of maps and $X$ in a particular class $C$ of compacta, then we will say that the branchpoint covering property holds for $\mathcal{F}$ on $\mathcal{C}$.

It is an immediate consequence of Whyburn's classical theorem on the lifting of dendrites under light open maps [8,p.188] that the branchpoint covering property holds for light open maps on compacta. In this paper we prove that the branchpoint covering property holds more generally for confluent and weakly confluent maps on certain classes of compacta. As a corollary we show that the weakly confluent image of a graph is a graph, thus generalizing a theorem of Whyburn [8,p.182].

I. Confluent maps on hereditarily arcwise connected compacta. Following Charatonik [1], we define a surjective map $f: X \rightarrow Y$ to be confluent if for each subcontinuum $A$ of $Y$, each component of $f^{-1}(A)$ is mapped by $f$ onto $A$. It follows from a theorem of Whyburn [8,p.148] that open maps on compacta are confluent.

Our first theorem shows that the branchpoint covering property holds for confluent maps on hereditarily arcwise connected compacta (i.e. compacta in which each subcontinuum is arcwise connected).

Received by the editors March 21, 1973 and, in revised form, October 16, 1974.

AMS (MOS) subject classifications (1970). Primary 54C10; Secondary 54F50.

Key words and phrases. Branchpoint, confluent map, weakly confluent map, hereditarily arcwise connected compactum, hereditarily locally connected continuum, graph. 
I.1. THEOREM. If $f$ is a confluent map from a hereditarily arcwise connected compactum $X$ onto a compactum $Y$, then $Y$ is hereditarily arcwise connected and $f$ covers the branchpoints of $Y$.

Proof. It is immediate that $Y$ is hereditarily arcwise connected since $f$ is (weakly) confluent and arcwise connectivity is a continuous invariant.

Now let $p$ be a branchpoint of $Y$ and let $T$ be a triod in $Y$ whose vertex is $p$. We shall find a triod $T^{\prime}$ in $f^{-1}(T)$ such that $f\left(T^{\prime}\right)=T$ and the vertex of $T^{\prime}$ covers $p$.

Label the endpoints of $T$ by $a, b$, and $c$. Choose a component $C_{1}$ of $f^{-1}(a b)$ ( $a b$ is the arc in $T$ with endpoints $a$ and $b$ ). Since $f$ is confluent, $f\left(C_{1}\right)=a b$. Hence we can pick $a^{\prime}$ and $b^{\prime}$ in $C_{1}$ so that $f\left(a^{\prime}\right)=a$ and $f\left(b^{\prime}\right)=b$. Let $a^{\prime} b^{\prime}$ be an arc in $C_{1}$ with endpoints $a^{\prime}$ and $b^{\prime}$ and pick $p^{\prime} \in a^{\prime} b^{\prime}$ so that $f\left(p^{\prime}\right)=p$. Now choose the component $C_{2}$ of $f^{-1}(p c)$ which contains $p^{\prime}$. Since $f\left(C_{2}\right)$ $=p c$, there is a point $c^{\prime}$ in $C_{2}$ with $f\left(c^{\prime}\right)=c$. Let $c^{\prime} p^{\prime}$ be an arc in $C_{2}$ from $c^{\prime}$ to $p^{\prime}$ and choose the first point $q$ of $c^{\prime} p^{\prime}$ such that $q \in a^{\prime} b^{\prime}$. Then since $a b \cap p c=\{p\}$, we have that $f(q)=p$ and so $T^{\prime}=c^{\prime} q \cup a^{\prime} b^{\prime}$ is the desired triod.

We remark that $\mathrm{I} .1$ fails if $X$ is required only to be arcwise connected, even if $f$ is monotone.

I.1 is helpful in classifying the confluent images of certain hereditarily arcwise connected compacta. A fan is a dendroid with one branch point [2].

I.2. Corollary. The confluent image of

$$
\left\{\begin{array}{l}
\text { an arc } \\
\text { a circle } \\
\text { a fan }
\end{array}\right\} \text { is } \quad\left\{\begin{array}{l}
\text { an arc } \\
\text { an arc or circle } \\
\text { a fan on an arc }
\end{array}\right\} \text {. }
$$

Proof. This follows from I.1 and the fact that the confluent image of a dendroid is a dendroid [1].

Contained in the proof of $\mathrm{I} .1$ is a triod covering theorem: each triod $T$ in $Y$ is the image of a triod $T^{\prime}$ in $X$ whose vertex is mapped to the vertex of $T$. Note that the obvious monotone map from an " $H$ " onto an " $X$ " shows that this fails for $n$-ods in general, $n>3$. However, in case $f$ is also light, then $n$-ods can be covered. In fact, we can state the following theorem, which does not seem to have been noted previously.

I.3. THEOREM. If $f$ is a light confluent map from a compactum $X$ onto a compactum $Y$, then for each dendrite $D$ in $Y$, and each point $x \in f^{-1}(D)$, there is a dendrite $D^{\prime}$ in $X$ such that $x \in D^{\prime}$ and $f$ maps $D^{\prime}$ homeomorphically onto $D$.

Proof. (We thank the referee for supplying this argument.) Let $f_{1}$ $=\left.f\right|_{f^{-1}(D)}$. Then $f_{1}: f^{-1}(D) \rightarrow D$ is onto and confluent $[1, \mathrm{p} .213]$, and hence it is locally confluent [4, Theorem 2]. The range space of $f_{1}$ being locally connected, we conclude from Corollary 5.2 of [5] that $f_{1}$ is an OM-mapping. But since $f_{1}$ is also light, it follows that $f_{1}$ is open. Now Whyburn's Theorem $2.4[8]$ applies.

II. Weakly confluent maps on hereditarily locally connected continua. A map $f$ from $X$ onto $Y$ is said to be weakly confluent [7] if for each subcontinuum $A$ of $Y$, at least one of the components of $f^{-1}(A)$ maps onto $A$ under $f$. Recent 
results indicate that, in general, weakly confluent maps form a much larger class than confluent maps. For example, any mapping of a continuum into a chainable continuum is weakly confluent [7], while no mapping from a hereditarily indecomposable continuum onto an arc is confluent (because hereditary indecomposability is invariant under confluent maps). Now, as we shall show by example in $\S \mathrm{III}$, the branchpoint covering property fails to hold for weakly confluent maps on hereditarily arcwise connected compacta. However, if we restrict our attention to certain hereditarily locally connected continua (continua in which each subcontinuum is locally connected), then we can retrieve the branchpoint covering property for weakly confluent maps.

II.1. THEOREM. If $f$ is a weakly confluent map from a hereditarily locally connected continuum $X$ onto a continuum $Y$, then $Y$ is hereditarily locally connected and the branchpoints of $Y$ are covered by the closure of the branchpoints of $X$.

Proof. The hereditary local connectedness of $Y$ is immediate since $f$ is weakly confluent and the continuous image of a locally connected continuum is locally connected.

Now let $p$ be a branchpoint of $Y$ and let $U$ be an open set containing $p$. In order to show that $p$ is covered by the closure of the branchpoints of $X$, it suffices to find a branchpoint $q$ of $X$ such that $f(q) \in U$. Choose a triod $T$ in $U$ having $p$ as its vertex. We will find $q$ in $f^{-1}(T)$.

Label the endpoints of $T$ by $a, b$, and $c$. On the arc $a p$ in $T$, choose a sequence of points $a_{i}$ converging to $a_{0} \neq p$ such that $a<a_{i}<a_{i+1}<p$ for each $i$. Choose similar sequences $b_{i} \rightarrow b_{0} \neq p$ and $c_{i} \rightarrow c_{0} \neq p$ in the arcs $b p$ and $c p$ respectively. Denote by $T_{i}$ the subtriod of $T$ with endpoints $a_{i}, b_{i}, c_{i}$. Since $f$ is weakly confluent, we can find subcontinua $A_{i}$ in $f^{-1}(T)$ with $f\left(A_{i}\right)=T_{i}$. Since $f^{-1}(T)$ is compact, we can assume without loss of generality that the sequence $A_{i}$ converges in the exponential topology to a continuum $A_{0}$ in $f^{-1}(T)$. Now since $f$ is continuous, $f\left(A_{0}\right)=T_{0}$ [3]. Consequently, there is an $\epsilon>0$ such that the diameter of $A_{i}$ is greater than $\epsilon$ for sufficiently large $i$. Using [8,p.92] only finitely many components of $f^{-1}(T)$ have diameter greater than $\epsilon$, and, hence, we may assume that all of the continua lie in the same component $C$ of $f^{-1}(T)$. We will find $q \in C$.

Suppose $C$ contains no branchpoints. Then $C$ is a locally connected continuum with no branchpoints and so must be either an arc or a circle. In either case, it is clear that for each $i, A_{i} \backslash A_{i+1}$ has at most two components and that as $i$ gets large, the diameters of these components approach 0 . Let $\epsilon_{1}>0$ be chosen smaller than the diameter of each of the arcs $a_{0} b_{0}, b_{0} c_{0}$, and $c_{0} a_{0}$ of $T_{0}$. Choose $\delta>0$ so that for each subset $K$ of $C$ of diameter less than $\delta$, the diameter of $f(K)$ is less than $\epsilon_{1}$. Now choose $i$ so large that the components of $A_{i} \backslash A_{i+1}$ have diameter less than $\delta$. Since $A_{i} \backslash A_{i+1}$ has at most two components and $a_{i}, b_{i}, c_{i}$ lie in $f\left(A_{i}\right) \backslash f\left(A_{i+1}\right)=T_{i} \backslash T_{i+1}$, at least two of $f^{-1}\left(a_{i}\right), f^{-1}\left(b_{i}\right)$, and $f^{-1}\left(c_{i}\right)$ must meet the same component of $A_{i} \backslash A_{i+1}$. But if $C^{\prime}$ is a component of $A_{i} \backslash A_{i+1}$ containing $a_{i}^{\prime} \in f^{-1}\left(a_{i}\right)$ and $b_{i}^{\prime} \in f^{-1}\left(b_{i}\right)$, then $f\left(C^{\prime}\right) \supseteq a_{i} b_{i}$ $\supseteq a_{0} b_{0}$ and so has diameter greater than $\epsilon_{1}$. From this contradiction we conclude that $C$ must contain a branchpoint $q$.

II. 1 can be generalized somewhat. Let $\mathcal{C}$ denote the class of all compacta $X$ 
having the property that for each closed subset $A$ of $X$ and each $\epsilon>0$ only finitely many components of $A$ have diameter exceeding $\epsilon$. Note that by [8,p.92] closed subsets of hereditarily locally connected continua are members

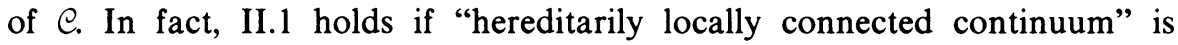
replaced by "a member of $\mathcal{C}$ " in the hypothesis.

II.2. COROLlARY. The branchpoint covering property holds for weakly confluent maps on hereditarily locally connected continua whose branchpoints form a closed set.

It follows from II.2 that if $Y$ is a weakly confluent image of a hereditarily locally connected continuum with $n$ branchpoints $(n<\infty)$, then $Y$ has no more than $n$ branchpoints. Thus we have a convenient tool for determining the weakly confluent images of certain hereditarily locally connected continua.

II.3. COROLLARY. The weakly confluent images of

$$
\left\{\begin{array}{l}
a \text { arc } \\
\text { a circle } \\
a \text { fan }
\end{array}\right\} \text { consist precisely of }\left\{\begin{array}{l}
a n \text { arc and a circle } \\
\text { an arc and a circle } \\
a \text { fan and an arc }
\end{array}\right\} .
$$

Proof. Any weakly confluent image of an arc or a circle must be a locally connected continuum with no branchpoints, hence an arc or a circle. The map $f:[0,1] \rightarrow S^{\prime}$ given by $f(t)=e^{4 t \pi i}$ is weakly confluent. Also, retractions are weakly confluent.

In order to prove that the weakly confluent image of a graph is a graph we need the following two lemmas. The first is used by Whyburn and is stated without proof.

II.4. Lemma. Suppose $f$ is a light map from a compactum $X$ onto a compactum $Y$. Then for each $\epsilon>0$ there is a $\delta>0$ such that if $A$ is a subcontinuum of $Y$ of diameter less than $\delta$, then each component of $f^{-1}(A)$ has diameter less than $\epsilon$.

II.5. LEMMA. Suppose $f$ is a light weakly confluent map from a graph $X$ onto a compactum $Y$. Then if $p$ is the vertex of an $n$-od in $Y$, then there is an $n$-od in $X$ with vertex $q$ such that $f(q)=p$.

Proof. Choose $\epsilon>0$ to be less than the minimum distance between distinct branchpoints of $X$ and also less than the minimum diameter of a circle in $X$. Apply II.4 to obtain a $\delta>0$. Now if $p$ is the vertex of an $n$-od in $Y$, it is the vertex of an $n$-od $N$ of diameter less than $\delta$. By the choice of $\epsilon$, each component of $f^{-1}(N)$ contains at most one branchpoint in $X$ and no circle in $X$ and so is a point, an arc, or an $m$-od for some $m \geqslant 3$.

Label the endpoints of $N$ by $a_{1}, a_{2}, \ldots, a_{n}$. For each $i=1, \ldots, n$, choose a sequence $a_{i j}$ in the arc $a_{i} p$ of $N$ converging to a point $a_{i 0} \neq p$ so that $a_{i}<a_{i j}<a_{i(j+1)}<p$ for each $i, j$. Denote by $N_{j}$ the sub- $n$-od of $N$ with endpoints $a_{1 j}, a_{2 j}, \ldots, a_{n j}$. Let $A_{j}$ be a subcontinuum of $X$ such that $f\left(A_{j}\right)$ $=N_{j}$. As in the proof of II.1, we can assume that all of the continua $A_{j}$ lie in the same component $C$ of $f^{-1}(N)$ and converge to a subcontinuum $A_{0}$ of $C$. By construction, $C$ is an arc or an $m$-od for some $m \geqslant 3$. We claim that $m \geqslant n$. For if $m<n$, then as II.1, $j$ can be chosen so that the components of 
$A_{j} \backslash A_{j+1}$ are all mapped by $f$ to subsets of $N_{j} \backslash N_{j+1}$ having diameters less than the minimum of the diameters of the arcs $a_{r 0} a_{s 0}, r \neq s$, in $N_{0}$. However, by construction, $N_{j} \backslash N_{j+1}$ has $n$ components and $A_{j} \backslash A_{j+1}$ has at most $m$ components. Consequently, at least two of the sets $f^{-1}\left(a_{i j}\right), i=1, \ldots, n$, must meet the same component of $A_{j} \backslash A_{j+1}$. From this impossibility we conclude that $m \geqslant n$. Now since $\epsilon$ can be chosen arbitrarily small, it follows that for some choice of $\epsilon$, the vertex $q$ of $C$ is such that $f(q)=p$.

\section{II.6. THEOREM. The weakly confluent image of a graph is a graph.}

Proof. Let $f: X \rightarrow Y$ be a weakly confluent map from the graph $X$ onto $Y$. Form the monotone-light factorization of $f, f=l \circ \mathrm{m}$. Then the middle space $X^{\prime}=m(X)$ is a graph (this follows from the fact that the monotone image of an arc is an arc or a point). Let $n$ be the highest order of the branchpoints of $X^{\prime}$. Suppose $Y$ is not a graph. Then there is an $(n+1)$-od in $Y$. But since $l$ is a light weakly confluent map, II.5 asserts the existence of an $(n+1)$-od in $X^{\prime}$, contradicting the choice of $n$. This completes the proof.

Question 1. Given a graph $X$, is there an algorithm for listing those graphs which are weakly confluent images of $X$ ? Note that there are only finitely many such images.

Question 2. Is every graph the weakly confluent image of a finite tree?

III. Examples. In this section, we construct two examples which show that the hypothesis of II.1 that $X$ be a hereditarily locally connected continuum cannot be weakened to hereditary arcwise connectedness nor can the conclusion be strengthened by removal of the word "closure".

III.1. EXAMPLE. A weakly confluent map of the Cantor fan onto a a triod which takes the vertex of the fan to an endpoint of the triod.

Let $E$ denote the Cantor middle third set and let $T$ be the standard triod $\{(x, y) \mid y=0$ and $|x| \leqslant 1$ or $x=0$ and $0 \leqslant y \leqslant 1\}$. Let $g$ be a continuous function from $E$ onto the unit interval $[0,1]$.

Now define a function $F: E \times[-2,2] \rightarrow T$ by

$$
F(x, y)= \begin{cases}(y+1,0) & \text { if }-2 \leqslant y \leqslant-1, \\ (0, \min \{g(x), 1-|y|\}) & \text { if }|y| \leqslant 1, \\ (y-1,0) & \text { if } 1 \leqslant y \leqslant 2 .\end{cases}
$$

It is easily checked that $F$ is well defined and onto $T$. Also $F$ is continuous because the restriction of $F$ to each of the three closed sets $-2 \leqslant y \leqslant 1$, $|y| \leqslant 1$, and $1 \leqslant y \leqslant 2$ is continuous.

Claim. $F$ is weakly confluent.

Proof. Let $K$ be a subcontinuum of $T$. If $(0,0) \in K$, then there exist numbers $a, b, c$ with $0 \leqslant a, b, c \leqslant 1$ such that the endpoints of $K$ are $(-a, 0)$, $(0, b),(c, 0)$. Choose $e \in E$ such that $g(e)=b$ and consider the arc $A$ $=\{(e, y) \mid-a-1 \leqslant y \leqslant c+1\}$ in $E \times[-2,2]$. It is easily checked that $F(A)$ $=K$. If $(0,0) \notin K$ then $K$ is an arc not containing the vertex of $T$. Choose $e \in E$ with $g(e)=1$. Then one can find an arc $A$ in $\{e\} \times[-2,2]$ such that $f(A)=K$.

Now to construct the example, note that $F^{-1}(-1,0)=E \times\{-2\}$, and let $X$ 
be the quotient space of $E \times[-2,2]$ obtained by identifying $F^{-1}(-1,0)$ to a point.

Let $G$ be the map from $X$ onto $T$ induced by $F$. Then noting that $X$ is a copy of the Cantor fan and $G$ is weakly confluent, the example is complete.

III.2. REMARKS. If we form the monotone-light factorization of the map $G$, $G=L \circ M$, then it is easily seen that the middle space $X^{\prime}=M(X)$ is also a copy of the Cantor fan and so $L$ is a light weakly confluent map with the same property as $G$.

Lelek has asked whether the confluent image of a chainable continuum must be chainable [4]. McLean has shown that such an image must be treelike [6]. Of course, the weakly confluent image of a chainable continuum need not be tree-like (see II.3); however, it may be wondered if the weak confluent image of a chainable continuum must be chainable provided it is tree-like. The answer to this is no, for the map $F$ may be extended in a natural way to a weakly confluent map from the chainable continuum described by Kuratowski [3,p.191] onto the triod $T$.

III.3. EXAMPLE. A weakly confluent map from a dendrite onto a triod with the property that no branchpoint of the dendrite is mapped to the vertex of the triod.

Let $T$ be the triod with vertex $p$ and endpoints $a, b$, and $c$ as shown in Figure 1 .

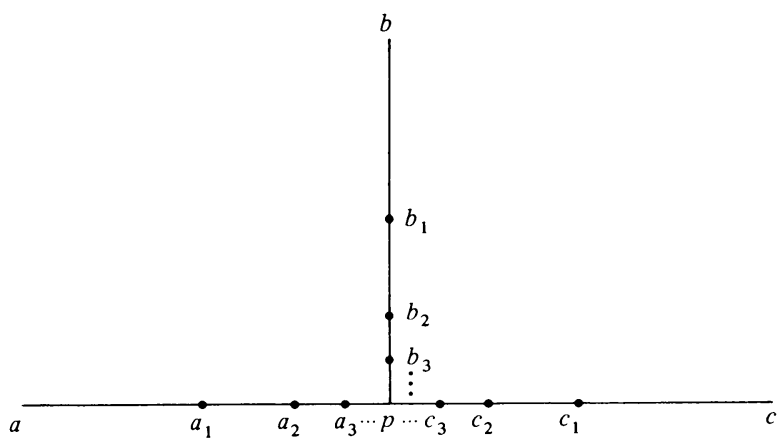

Figure 1

On each of the segments $a p, b p$ and $c p$ construct sequences $a_{i}, b_{i}$, and $c_{i}$ converging monotonically to $p$.

Now the domain $D$ of the mapping is pictured in Figure 2.

Certain points have been labelled by their image under the mapping. The arcs connecting two labelled points with no labelled points between them are to be mapped linearly onto the appropriate arc in Figure 1. This completely determines the mapping. Notice that it is light. One argues that it is weakly confluent by observing that if $A$ is a subcontinuum of $T$, one of whose endpoints lies outside the subtriod $T_{n}$ of $T$ with endpoints $a_{n}, b_{n}$, and $c_{n}$, then one can find in the subdendrite $D_{n}$ of $D$ consisting of the entire horizontal segment together with the $3 n$ vertical segments labelled $a_{1} c, a_{2} c_{1}, \ldots, a_{n} c_{n-1}$, $b_{1} a, b_{2} a_{1}, \ldots, b_{n} a_{n-1}, c_{1} b, c_{2} b_{1}, \ldots, c_{n} b_{n-1}$, a continuum mapping into $A$. 


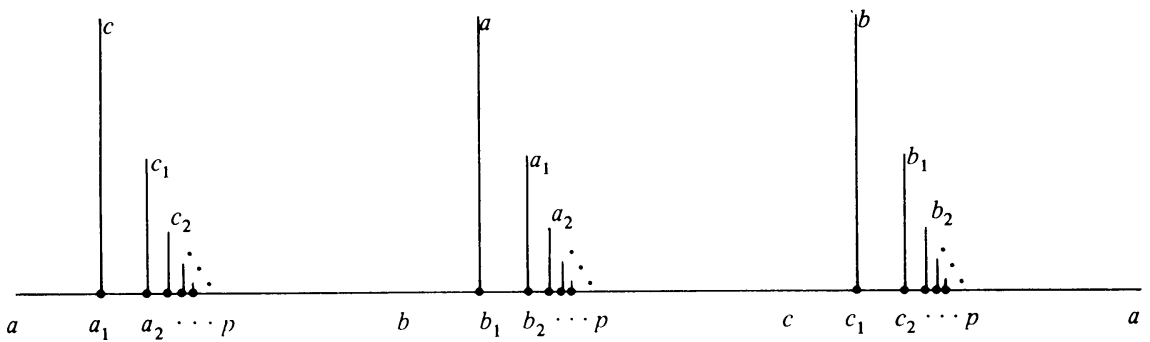

\section{FIGURE 2}

\section{REFERENCES}

1. J. J. Charatonik, Confluent mappings and unicoherence of continua, Fund. Math. 56 (1964), 213-220. MR 31 \# 723.

2. _- On fans, Dissertationes Math. Rozprawy Mat. 54 (1967), 39 pp. MR 37 \# 3528.

3. K. Kuratowski, Topology. Vol. 1, PWN, Warsaw; Academic Press, New York, 1966. MR 36 \#840.

4. A. Lelek, A classification of mappings pertinent to curve theory, Proc. Univ. of Oklahoma Topology Conf., Norman, Oklahoma, 1972.

5. A. Lelek and D. R. Read, Compositions of confluent mappings and some other classes of functions, Colloq. Math. 29 (1974), 101-112.

6. T. Bruce McLean, Confluent images of tree-like curves are tree-like, Duke Math. J. 39 (1972), 465-473. MR 46 \#4502.

7. D. R. Read, Confluent, locally confluent, and weakly confluent maps, Dissertation, University of Houston, Houston, Tex., 1972.

8. G. T. Whyburn, Analytic topology, Amer. Math. Soc. Colloq. Publ., vol. 28, Amer. Math. Soc., Providence, R.I., 1963. MR 32 \#225.

DEPARTMENT OF MATHEMATICS, UNIVERSITY OF KENTUCKY, LEXINGTON, KENTUCKY 40506 (Current address of C. A. Eberhart and J. B. Fugate)

Current address (G. R. Gordh, Jr.); Department of Mathematics, Guilford College, Greensboro, North Carolina 27410 